Comparison of molecular dynamics simulation methods for the study of grain boundary migration

This article has been downloaded from IOPscience. Please scroll down to see the full text article. 2013 Modelling Simul. Mater. Sci. Eng. 21045017

(http://iopscience.iop.org/0965-0393/21/4/045017)

View the table of contents for this issue, or go to the journal homepage for more

Download details:

IP Address: 130.179.231.12

The article was downloaded on 28/05/2013 at 14:48

Please note that terms and conditions apply. 


\title{
Comparison of molecular dynamics simulation methods for the study of grain boundary migration
}

\author{
M I Mendelev ${ }^{1}$, C Deng ${ }^{2}$, C A Schuh ${ }^{3}$ and D J Srolovitz ${ }^{4}$ \\ ${ }^{1}$ Division of Materials Sciences and Engineering, Ames Laboratory, Ames, IA, 50011 USA \\ 2 Department of Mechanical and Manufacturing Engineering, University of Manitoba, Winnipeg, \\ Manitoba, R3T5V6, Canada \\ ${ }^{3}$ Department of Materials Science and Engineering, Massachusetts Institute of Technology, \\ Cambridge, MA 02139, USA \\ ${ }^{4}$ Departments of Materials Science and Engineering and Mechanical Engineering and Applied \\ Mechanics, University of Pennsylvania, Philadelphia, PA 19104, USA \\ E-mail: mendelev@ameslab.gov
}

Received 15 January 2013, in final form 27 March 2013

Published 10 May 2013

Online at stacks.iop.org/MSMSE/21/045017

\begin{abstract}
In the present study, grain boundary (GB) mobility was determined by molecular dynamics (MD) simulations using two different techniques: the applied strain method and the adapted interface random walk method. The first method involves a driving force while the second method does not. Nevertheless, both methods led to essentially the same values of the GB mobility. This shows that the GB mobility is independent of the nature of the driving force, provided that it is low enough that the linear velocity-driving force relationship is properly sampled. The case studied here can be viewed as a validated reference case that can be used in future studies to test new techniques to determine the GB mobility. For this purpose we provide the full information about the interatomic potential we employed and the initial atomic configurations. Finally, we use the obtained results to discuss whether any existing MD simulation data agree with experimental data on pure metals.
\end{abstract}

S Online supplementary data available from stacks.iop.org/MSMSE/21/ 045017/mmedia

(Some figures may appear in colour only in the online journal)

\section{Introduction}

Grain boundary (GB) mobility is a fundamental property and is needed as input to models of microstructure evolution. Yet our understanding of this property even in pure metals is very limited. Experimental data are usually affected by impurities, which have important consequences on GBs even in trace concentrations [1]. On the other hand, studying clean GBs is not a problem when using an atomistic computer simulation, so it is not surprising that 
much of our understanding of GB mobility in pure metals has been gleaned from molecular dynamics (MD) simulations [2]. Several methods have been proposed to extract the GB mobility from MD simulations and a body of data has been collected (see [2] for a review). However, there are two key questions that have not been satisfactorily resolved by these results. The first is whether all MD simulation methods produce the same value of GB mobility (for the same system and particular GB). The second key question is whether any of the existing MD simulation data agree with experimental data in the pure metals studied, or whether such agreement can ever be achieved with existing simulation and experimentation approaches. The goal of the present study is to address the first key question which, in turn, allows some speculation about the second.

In order to address the first question, we note that there are two main classes of MD simulation approaches used to determine GB mobility. The first class involves application of a driving force to move the GB. For example, a strain applied to a bicrystal can induce a jump in strain energy across the boundary, leading to boundary migration [3,4]. The driving force in this case has a clear physical meaning and the simulation indeed models a real physical process. On the other hand, there are limitations associated with symmetric GBs for which an applied strain will not produce a driving force for GB migration. Therefore, an artificial energy method was proposed in [5]. In this method, an artificial energy is added to the potential energy based on the orientation of the nearest neighbor bonds. This artificial energy produces a driving force for GB migration, although there is some concern that it 'does not arise from a physical cause such as stress or a magnetic field but rather is applied synthetically' [5]. However, it was shown in [6] that this method can yield the same value of the GB mobility as the applied strain method.

MD simulation methods where the GB curvature is used as the source of a driving force $[7,8]$ represent a special case of the class of driven methods to extract the GB mobility. In this case, the so-called reduced mobility, which is the product of the GB mobility and stiffness (GB free energy plus its second derivative with respect to GB orientation), is extracted, averaged over all the boundary inclinations used in the simulation [7,8]. While the geometry of this method more directly matches that used in some experiments [9], it requires an independent assessment of the GB stiffness, which is nontrivial [2], to obtain the actual GB mobility. Therefore, the data produced by this method are difficult to compare with those obtained from different techniques.

The GB mobility, $M$, in the driven MD simulations (including the cases where the curvature is used as the driving force) is usually determined by assuming that the driving force is small such that linear kinetics prevails:

$$
V=M P
$$

where $V$ is the GB velocity and $P$ is the driving force. The reliability of the data obtained in this manner rests on the question of whether the velocity-driving-force relationship is indeed linear at the driving forces used in those MD simulations. It has been shown that at large driving forces, and especially at lower temperatures, the $V-P$ relationship is not linear $[4,10,11]$. Therefore, it is a necessary condition on the reliability of the driven methods that the $V-P$ relationship is indeed linear, or can be extrapolated into the linear regime, under the simulation conditions. In general, this requires the measurement of GB velocities over a range of driving forces. Unfortunately, this approach suffers some complications. First, there is usually uncertainty in the simulation data, and especially for historical data generated during a period of more limited computational power, the number and accuracy of the available data points may not support statistically meaningful conclusions about the linearity of the $V-P$ relationship. Second, even if the linearity is established, it can be very difficult to distinguish between the linear dependence corresponding to equation (1) and a different linear dependence, such as $V=\mu P+\beta$. In fact, in [11] it was shown that there can be two distinct linear regimes in the $V-P$ relationship, 
and these may be only subtly different in the values of $\mu$ and $\beta$ depending upon conditions (temperature, GB bicrystallography, etc). This means that the value of the slope obtained in the high driving-force regime, where a different ('ballistic' rather than 'diffusive' — see [11] for detailed discussion) mode of GB migration is probed, cannot necessarily be viewed as the traditional 'diffusive' GB mobility. The high driving-force regime is discussed extensively in [11] and interested readers are referred to that discussion rather than focusing on it here (this manuscript focuses on comparisons of simulation methods-especially in the experimentally meaningful, low driving-force regime). The authors of [11] showed that if too much data from the high driving-force regime are included when determining the activation energy for GB migration, the resulting value can be artificially small and lose its intended physical sense. This provides another possible contribution (in addition to the impurity effects discussed above) to the standing discrepancy between the activation energies cataloged by MD simulations and those reported in experiments, taking into account that the latter are usually significantly higher $[3,4,12]$.

The second class of methods to extract the GB mobility via MD simulations is based upon analysis of fluctuations of either the GB profile [13] or position [14]. While the derivations of the main equations used in these methods explicitly assume a linear $V-P$ relationship (equation (1)), these methods do not employ any driving force. Therefore, the results obtained using these methods seem to be the most relevant to experiments. However, these methods also require independent validation. For example, the authors of [13], in which the GB profile fluctuations were analyzed, state that the method based on the GB profile fluctuation analysis cannot be applied for the GBs where a cusp in the energy versus misorientation/inclination is expected or at low temperatures. Thus, it is desirable to compare the GB mobilities obtained using this fluctuation method with an independent approach (note that this fluctuation method provides an opportunity to extract the GB stiffness from the MD simulation which is a great challenge itself).

The authors of [14] explored fluctuations of the average GB position $p$, which varies with time $t$ as

$$
\left\langle\bar{p}^{2}\right\rangle=\frac{2 M k_{\mathrm{B}} T}{A} t
$$

where $k_{\mathrm{B}} T$ is the thermal factor and $A$ is the GB area. They came to the conclusion that the driven methods lead to mobilities that are close to an order of magnitude lower than those obtained using equation (2). Since some driven methods were shown to be in the linear $V-P$ regime in some simulations, only a rather complicated $V-P$ relationship that changes slope with $P$ can explain such a disagreement.

Thus, while the fluctuation methods are nominally extremely useful to the study of true GB mobility properties, there remain some points of clarity on how generally applicable they are and how their predictions compare to those of the driven methods. The authors of $[11,15]$ provided a first point of connection between these classes of methods by applying a bias force $P$ to the fluctuation method of [14], combining the driven and fluctuation approaches in a single method called the adapted interface random walk (AIRWalk). Through this approach, they were able to study somewhat slower GB velocities than in previous works and showed that there is a high driving-force regime in which the driven methods do not yield conventional 'diffusional' GB mobility data. Nonetheless, there remain many unresolved issues that center on the first key question raised above-whether all MD simulation methods can produce the same value of the mobility. For example, it is still not clear if the value of the GB mobility is independent of the nature of the applied driving force. One can argue that different driving forces can lead to different mechanisms of GB migration, and therefore different mobilities characterizing this migration. As noted in [2], this 'remains an open question' and the authors 
of [14] considered this as a possible explanation for the difference between the results of their simulation data and the previous driven simulation results. An attempt to investigate this issue was made in [16] using the Ising model. The authors of [16] concluded that the interface mobility does not depend on the nature of the driving force but it is not clear whether this is a general conclusion applicable to all GBs or it is valid only for interfaces in the simplistic Ising model.

Unfortunately, at present it is very difficult to compare the mobilities obtained using different methods because the various published studies come from different groups with different foci and are not designed to be compared with one another. They generally study different metals, different GBs and/or employ different interatomic potentials. There are just a few examples where direct comparisons are made $[6,11,14]$ and no clear picture has yet emerged. We believe that it would be a tremendous benefit to the field to have a reference case suitable for broad comparisons of the methods. This reference case could be used to explore the conditions under which different methods are applicable, and could help evaluate the strengths and limitations of both old and new methods (e.g. see the discussion in [17, 18]). In the present paper, we propose such a reference case (we choose a particular GB in Ni) and investigate the GB mobility using two different methods, one driven and one a fluctuation method. We show that there are conditions under which both methods can indeed yield the same mobility and also provide some insight into the question of under what conditions disagreement may emerge.

The remainder of the paper is organized as follows. First, we introduce the proposed reference case and briefly describe the simulation methods we used to determine the GB mobility: the AIRwalk technique and elastically driven GB migration. Next, we provide GB mobility data obtained using these two independent methods. Finally, we discuss the results and explore their implications for the overarching questions delineated above.

\section{MD simulation methods}

\subsection{The reference case}

A reference case should be chosen such that any, or at least most, methods for determining GB mobility via MD simulation can be applied. The applied strain method proposed in [4] can only be used in cases where the GB is asymmetric. At the same time, a highly symmetric boundary near a deep energy cusp may not be amenable to study by some of the fluctuation methods, and may also be a 'special case' that does not properly present all of the most important physical issues germane to GB migration. Accordingly, we propose as a reasonable reference case the $\Sigma 5\left[\begin{array}{lll}0 & 1 & 0\end{array}\right]$ tilt boundary, inclined such that the boundary plane corresponds to (llll $\left.\begin{array}{lll}1 & 0\end{array}\right)$ and $\left(\begin{array}{lll}1 & 0 & 1\end{array}\right)$ surfaces in the upper and lower crystals, respectively. This GB is shown in figure 1, and is perpendicular to the $z$-axis. Our simulation cell also uses two free surfaces perpendicular to the $z$-axis and periodic boundary conditions in the directions parallel to the nominal boundary plane and the free surfaces.

The GB (and the simulation cell set-up) in figure 1 can be used to compare, e.g., different potentials and methods. Since our focus is on a comparison of driven versus fluctuation methods, we elected to use a single, semi-empirical potential for all of the present simulations; we employed that developed for $\mathrm{Ni}$ in $[19,20]$. Although the melting temperature for this potential is $1265 \mathrm{~K}$ (obtained from the liquid-solid co-existence method [21]), which is much lower than the actual melting temperature for $\mathrm{Ni}$ (1728 K [22]), this potential permits direct comparison with a prior study of GB mobility in [11]. In addition, it uses a rather short cut-off radius $(4.32 \AA)$ which accelerates computations. While we would not propose that results obtained using this older potential be directly compared with experiments on $\mathrm{Ni}$, this choice seems to be reasonable for developing the reference case. 
(a)

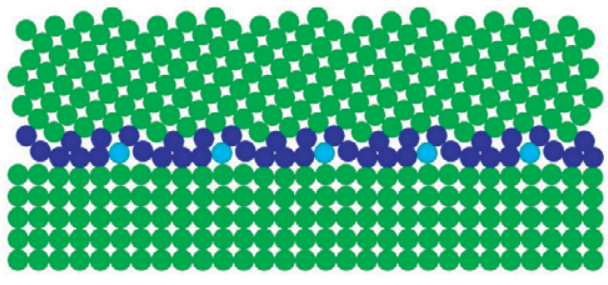

(b)

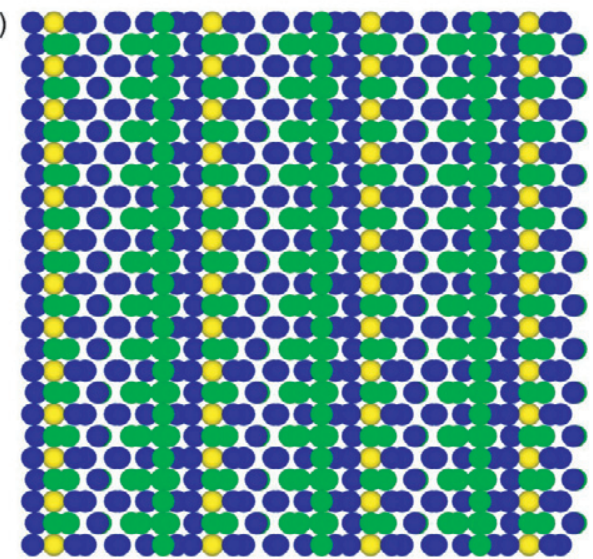

$\uparrow:[10 \overline{7}]$

$\underset{y:[0 \overline{1} 0]}{\longrightarrow} x:[701]$

A: $[101]$

$\underset{y:[010]}{\longrightarrow} x:[101]$

Figure 1. (a) The Atomistic configuration and plane indices of the simulated grain boundary; (b) Atomistic view of the grain boundary plane at $0 \mathrm{~K}$. Atom colors correspond to local atomic symmetry.

We started by determining the temperature dependence of the fcc lattice parameter using the procedure described in [23]. Our results can be summarized by the following polynomial fit, which returns a lattice parameter in units of $\AA$ when temperature is expressed in $\mathrm{K}$ :

$$
\begin{aligned}
a=3.524248 & +5.27256 \times 10^{-5} T+1.32804 \times 10^{-8} T^{2}-6.69173 \times 10^{-11} T^{3} \\
& +8.62013 \times 10^{-14} T^{4}-2.12751 \times 10^{-17} T^{5} .
\end{aligned}
$$

For the determination of the GB mobility, we employed a $\sim 4 \times 4 \times 16 \mathrm{~nm}$ simulation cell, accommodating 28000 atoms. A starting atomic configuration was constructed using the equilibrium lattice parameter given by equation (3) at $800 \mathrm{~K}$. The GB plane was perpendicular to the $z$-axis and initially located $2.5 \mathrm{~nm}$ from the top surface. The system was equilibrated at $T=800 \mathrm{~K}$ during $10000 \mathrm{MD}$ steps (the MD step is $2 \mathrm{fs}$ ). Next, the system was heated to $1000 \mathrm{~K}$ and held for $10000 \mathrm{MD}$ steps and then cooled to $800 \mathrm{~K}$. This yields a second initial configuration for the migration simulations. Repeating this process two additional times, we obtained a total of four separate initial atomic configurations for use in the migration studies. All of these initial atomic configurations along with the semi-empirical potential can be found in [24] in the LAMMPS format (version: 2012April10th) [25].

In the case of a driven method simulation, it is convenient for the GB to move as great a distance as possible. Therefore, the GB was initially located close to the top free surface and the MD simulation ran until the GB nearly reached the bottom free surface. In the case of fluctuation methods, the GB can move in either direction, and therefore it is convenient to start from initial models in which the GB is located in the middle of the simulation cell. Accordingly, to get the initial atomic configurations for our fluctuation simulations at $T=800 \mathrm{~K}$, a tensile biaxial strain of $1.5 \%$ was applied to each of the 4 initial models described above and the simulation was stopped when the GBs were close to the center of the simulation 
cell (after $5 \mathrm{~ns}$ ). The initial atomic configurations used at higher temperatures were obtained from these $T=800 \mathrm{~K}$ initial configurations by appropriate rescaling of the lattice parameter and equilibration over $10000 \mathrm{MD}$ steps.

We used two methods to determine the GB position, $p$. In the first, we determined the GB position from the maximum in the potential energy profile in the $z$-direction (normal to the nominal GB plane). The approximation inherent to this approach leads to an uncertainty in the GB position of $\sim 1 \AA$, which may lead to an error in the GB velocity not larger than $1 \%$ if the GB moves by $100 \AA$. Thus this method is suitable for the simulation of the GB migration under applied biaxial strain (see section 2.2) where the GB moved by $\sim 80 \AA$ during the MD simulation. The average GB velocity was determined from the slope of the $p$ versus $t$ dependence.

For fluctuation methods such as the AIRWalk method used in this work (see section 2.3), the GB displacement is smaller and a more precise method to determine the GB displacement is necessary. Since the AIRWalk method does not actually require determination of the GB position itself, we used a method to determine the GB displacement based on an order parameter depending on the local lattice orientation, as described in [5]. This order parameter is 0 for atoms at perfect lattice sites in one grain, 1 for atoms at perfect lattice sites in the other grain, and between 0 and 1 for atoms close to the GB plane. The GB displacement, $\Delta p$, can be obtained from the change of the average order parameter of the system, $\Delta \chi$, according to:

$$
\Delta p(t)=L_{z}^{\prime} \cdot \Delta \chi(t)
$$

where $L_{z}^{\prime}$ is the length of the simulation cell in the $z$-direction, excluding the free surfaces. The top and bottom free surfaces and the adjacent few atomic layers were not included in the determination of the average order parameter of the system.

\subsection{Simulation of $G B$ migration driven by applied biaxial strain}

In order to drive the GB at $T=800 \mathrm{~K}$, we applied a balanced biaxial strain in the directions parallel to the nominal boundary plane $\left(\varepsilon_{x x}=\varepsilon_{y y}=\varepsilon ; \varepsilon_{x y}=0\right)$. The strain was varied from $0.2 \%$ to $2 \%$ in the case of tension and from 0.5 to $2 \%$ in the case of compression. The first $10000 \mathrm{MD}$ steps (20 ps) were not used to determine the boundary velocity. Subsequently, the mean position, $\mathrm{p}$, of the GB position was recorded as a function of time and the boundary velocity determined from the slope of the $p-t$ plot.

In order to determine the driving force on the GB, separate simulations were performed. Simulation cells containing only one of the two grains (from above or below the boundary) were subject to the same strains as in the migration study and the stresses were determined using the standard virial expressions. The stress versus strain results are reported in figure 2. These dependences show deviations from linearity that are well characterized by a parabolic correction (at least over the strain regime explored) as

$$
\sigma_{x x}+\sigma_{y y}=\lambda_{1} \varepsilon+\lambda_{2} \varepsilon^{2} .
$$

The driving force is the difference between the free energies of the top and bottom grains per unit volume, and was calculated as

$$
P=\frac{1}{2} \Delta \lambda_{1} \varepsilon^{2}+\frac{1}{3} \Delta \lambda_{2} \varepsilon^{3},
$$

where $\Delta \lambda$ is the difference between the $\lambda$ coefficients for the top and bottom grains. Thus the definition of the driving force accounts for deviations from linear elasticity. It is important to note that while the driving force has the same units as a stress (energy per volume or GPa), the absolute value of the driving force is much smaller than the stress values (see figure 2). The maximum driving force used in the present work did not exceed $0.055 \mathrm{GPa}$. 

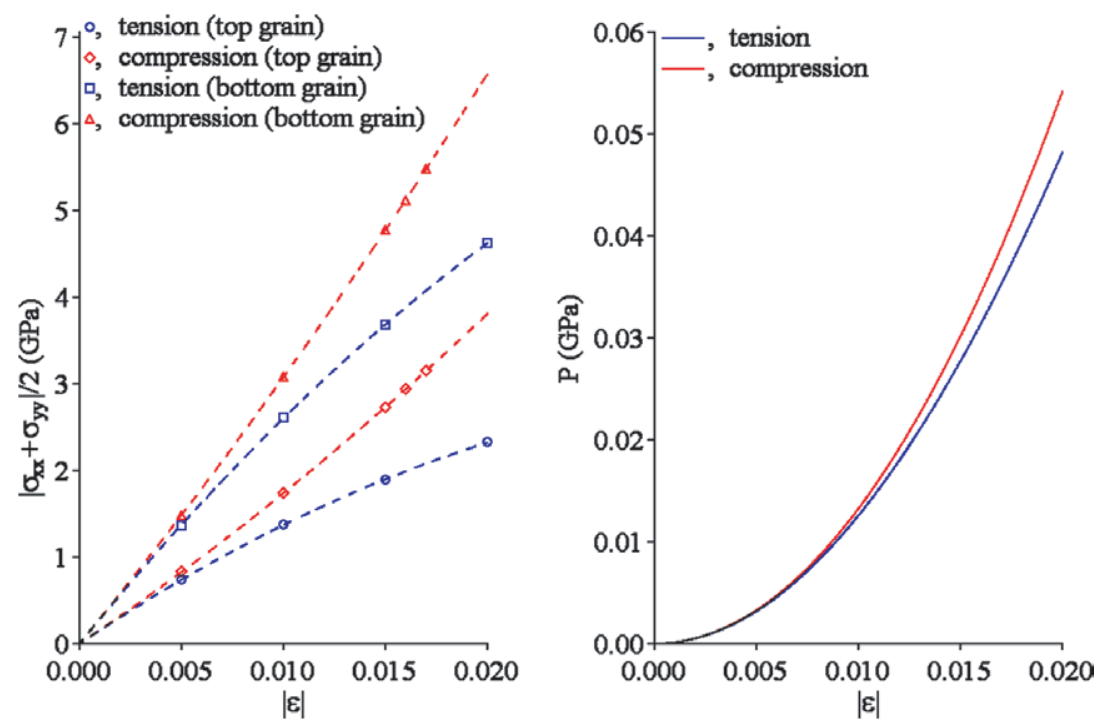

Figure 2. The bulk stresses and driving force as functions of the applied strain at $T=800 \mathrm{~K}$.
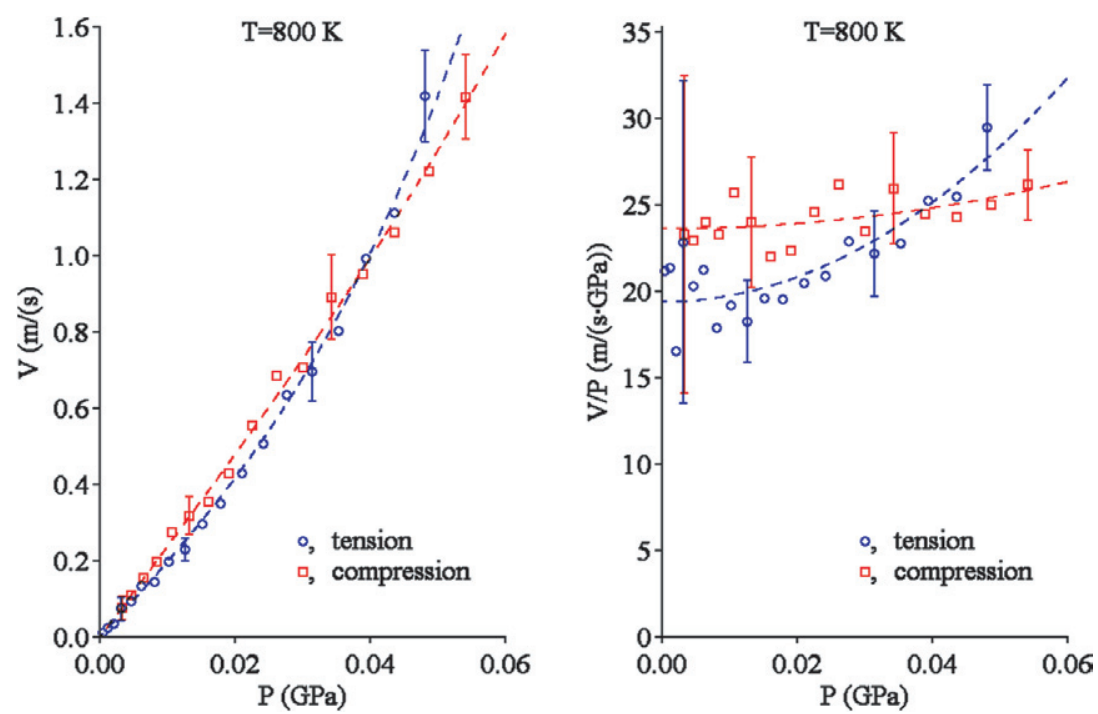

Figure 3. Grain boundary velocity as a function of driving force at $T=800 \mathrm{~K}$. The dashed lines correspond to the best fit according to equation (8). Typical uncertainties are shown for a few select points. The plot on the right shows the same data as in that on the left, but in the $V / P$ versus $P$ coordinates in accordance with equation (8).

The $V-P$ relationships for the tensile and compressive applied strains are shown in figure 3. All data points were averaged over four independent MD simulations. The same four independent MD simulations allowed us to estimate typical error bars, as shown in figure 3 for several typical data points. Examination of this figure shows that both the compression and tension studies yield similar results. However, at low driving forces, $V / P$ data points in compression are on average 18\% higher than those obtained in tension. At the highest driving forces studied, the situation is reversed. At low driving force, the error bars are much 
larger than the difference between the $V / P$ data in the compression and tension series. At the largest driving forces, the difference between the $V / P$ data in the compression and tension series may be attributed to the effect of stress on the mobility itself, as could be described in terms of an activation volume in the mobility. (While it would be possible to correct for these small effects, the general agreement between the tensile and compressive data over the range of studied driving forces in figure 3 suggests that both these effects can be neglected for the purposes of the subsequent discussion.)

As noted in the Introduction, it is important to the analysis of the driven GB migration data that the $V-P$ relationship is linear as in equation (1). Any simulation data corresponding to the non-linear $V-P$ regime should be excluded when the GB mobility is determined. However, as shown in [11], the transition is very gradual and varies with temperature, GB type, etc, so it is not clear how to define a 'critical driving force' below which the data are guaranteed to lie in the linear regime for diffusional GB motion. One approach to this problem would be to try to explicitly separate linear from higher-order terms in $P$. Since we expect that $V(-P)=-V(P)$, only odd-order corrections are required, and taking only the first of these we have

$$
V=M P+C P^{3}
$$

where $C$ is a constant which may depend on the type of the driving force.

In principle, equation (7) could be directly used to extract the mobility from the simulation data by least-squares fitting (which would isolate the linear contribution). However, in that case, the higher driving-force data would have an inappropriately large weight relative to the more relevant low driving-force data, simply because the velocity values are larger at higher driving forces. To remove this bias, we rearrange equation (7) as

$$
\frac{V}{P}=M+C P^{2}
$$

plot $V / P$ versus $P$ (as shown in the right plot in figure 2), and fit the intercept to obtain the mobility $M$. Using this method, we obtained $M_{\text {tens }}=19.4 \pm 3.4 \mathrm{~m} \mathrm{~s}^{-1} \mathrm{GPa}^{-1}$ in the tension series and $M_{\text {comp }}=23.6 \pm 1.1 \mathrm{~m} \mathrm{~s}^{-1} \mathrm{GPa}^{-1}$ in the compression series. The true value of the mobility should be between these values. By simple averaging of these values, we find $M=21.5 \mathrm{~m} \mathrm{~s}^{-1} \mathrm{GPa}^{-1}$.

\subsection{Determination of the GB mobility from the AIRWalk method}

For each of the four initial boundary structures, we performed four independent AIRWalk method simulations (different random velocity initialization) for times up to $25 \mathrm{~ns}$ at $T=800 \mathrm{~K}$. The data collected during the first $25 \mathrm{ps}$ were not used to determine the GB velocity.

In order to extract the GB mobility, the variance of the GB displacement, $\left\langle\bar{p}^{2}\right\rangle$, needs to be measured as a function of time according to equation (2). For each of the 16 simulations of $25 \mathrm{~ns}$ duration, the data were split into 25 groups of $1 \mathrm{~ns}$ each, equivalent to 400 independent simulations of $1 \mathrm{~ns}$ duration that can be processed according to the AIRWalk method [15]. Specifically, at any particular time $t,\left\langle\bar{p}^{2}\right\rangle$ was extracted by fitting the GB displacement from 400 simulations to a cumulative distribution function $f(x)$ [15] (see the inset in figure 4 for $t=500 \mathrm{ps}$ as an example). The enhanced statistics used in the AIRWalk method permits finer precision than does straight averaging of the measured quantities in the original interface random walk method [14]. The relationship between $\left\langle\bar{p}^{2}\right\rangle$ and $t$ for the 400 simulations is plotted in figure 4; clearly there is a linear relationship between these two quantities as predicted by equation (2). The resulting value of GB mobility, $M=21.2 \pm 2.0 \mathrm{~m} \mathrm{~s}^{-1} \mathrm{GPa}^{-1}$, is in agreement with the value obtained in the applied strain method. 


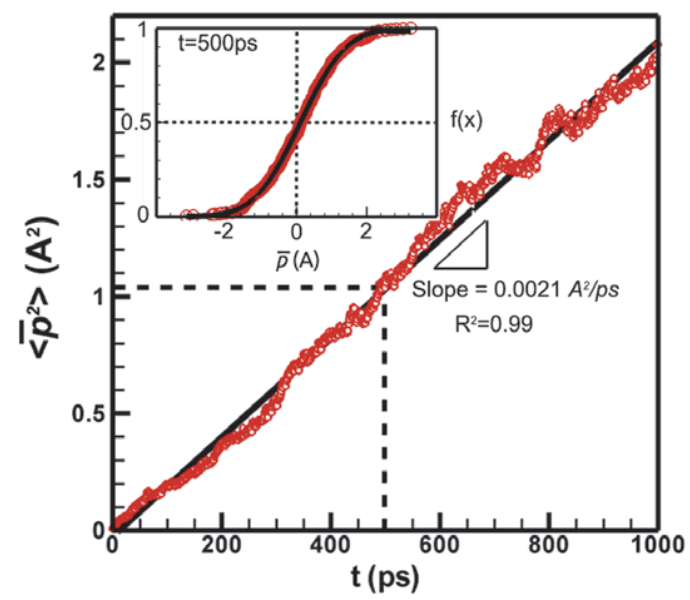

Figure 4. The variance of GB displacement $\left(\left\langle\bar{p}^{2}\right\rangle\right)$ as a function of time $(t)$ with no applied driving force. The solid line is a linear fit, while the dashed line indicates the variance value $\left(\left\langle\bar{p}^{2}\right\rangle=1.05 A^{2}\right)$ at $t=500 \mathrm{ps}$, corresponding to the distribution as indicated by the inset. The inset is the cumulative distribution of GB displacements from 400 independent simulations at $t=500 \mathrm{ps}$. The solid line in the inset is a theoretical prediction of the distribution, and the dashed lines are added as guides to the eye.

\subsection{Temperature dependence of the GB mobility}

In order to determine the temperature dependence of the GB mobility, we first simulated GB migration at $T=1200 \mathrm{~K}$ using the same procedure as described in section 2.2. The $V-P$ relationship for the case of the applied tensile biaxial strain is shown in figure 5. Examination of this figure shows that the $V-P$ relationship is linear when the driving force is smaller than $0.015 \mathrm{GPa}(\varepsilon=1.3 \%)$. Analysis of the data obtained at $T=800 \mathrm{~K}$ (see figure 2) also shows that the $V-P$ relationship is linear when the driving force is smaller than $0.015 \mathrm{GPa}(\varepsilon=1.1 \%)$. Thus, in order to determine the GB mobility at other intermediate temperatures $(900,1000$ and $1100 \mathrm{~K}$ ), we used tensile and compressive strains of $1 \%$. At all temperatures, four independent MD simulations were performed. The mobility was determined as the average of $V / P$ for the tensile and compressive series data. The results are shown in figure 6 . The same figure also shows the mobility data obtained from the AIRWalk method at the same temperatures. The mobilities determined by the two methods are in agreement.

The data in figure 6 do not fall exactly on a line and hence are not exactly Arrhenius. Yet, a fit to an Arrhenius relation is not unreasonable, and assuming Arrhenius behavior, the activation energy obtained from the applied strain method is $E_{\text {strain }}=0.45 \pm 0.03 \mathrm{eV} /$ atom, while that obtained from the AIRWalk method is $E_{\text {AIRWalk }}=0.44 \pm 0.03 \mathrm{eV} /$ atom. The agreement between the results from the two methods is well within the error bars.

\section{Discussion and conclusions}

In the present study, GB mobility was determined using two different techniques: a driven motion method (applied strain) and a fluctuation method. The first method involves the application of a driving force while the second involves no applied driving force. We demonstrate that both methods lead to the same values of the GB mobility. This addresses the first key question from the introduction and indicates that GB mobility is not affected by 


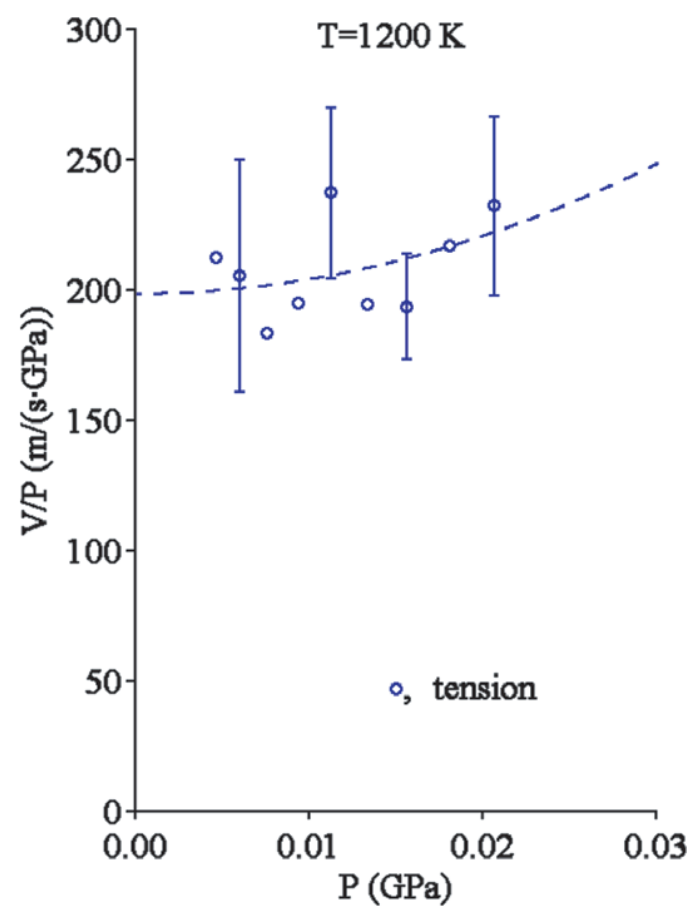

Figure 5. Grain boundary velocity as function of driving force at $T=1200 \mathrm{~K}$. The dashed lines correspond to the best fit to equation (8). The error bars were determined as in figure 3 .

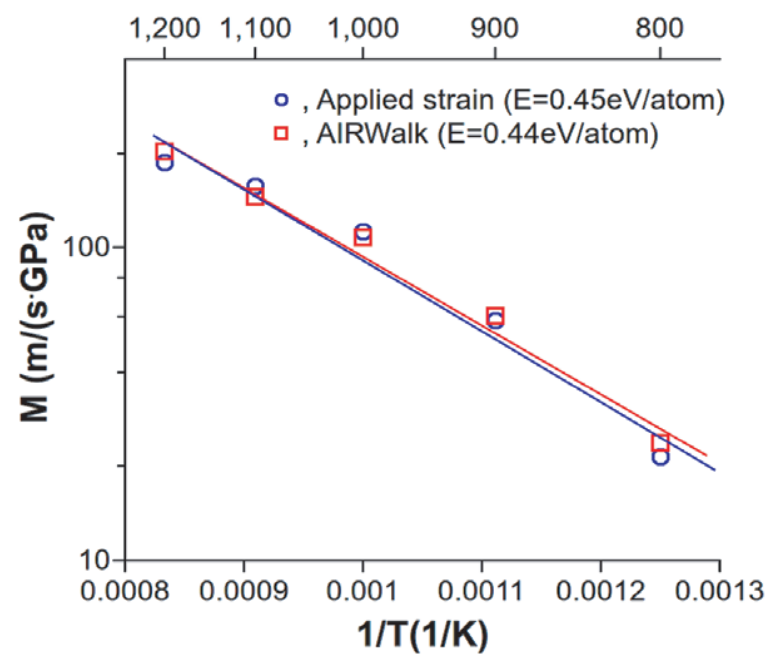

Figure 6. Temperature dependence of the GB mobility.

an applied driving force, provided that the driving force is sufficiently small that the diffusive mode of GB migration is sampled, with a linear relationship between the boundary velocity and the driving force. While the present results are not conclusive proof that GB mobility is independent of the nature of the driving force, it does suggest such an independence. This result demonstrates that fluctuation methods and applied driving-force methods can both be employed, provided that each is performed with sufficient care. Since the present results have 
been validated with very different approaches, we suggest that the present case (grain boundary type and interatomic potential) be employed as a standard test case for newly developed techniques for extracting grain boundary mobility from atomistic simulations. For this purpose, we provide [24] all of the necessary simulation details (including the interatomic potential and initial atomic configurations) for others to employ in such a standard test.

The present study reiterates that when using a driven method, it should not be assumed that the simulation probes the linear regime where diffusional boundary motion occurs. In the way that they are sometimes used, driven methods rely on the fact that the $V-P$ relationship is exactly linear up to the driving forces used in the simulation. Our results confirm that this should be checked explicitly every time driven methods are used. For example, it was shown in [11] that it is possible that the $V-P$ relationship is linear at high temperatures at the driving forces used in the simulation but it is not linear at low temperature. This can lead to considerable overestimation of the obtained values of the GB mobility at low temperatures and underestimation of the activation energy.

We now discuss the second key question in the Introduction; i.e., whether there is scope for finding agreement between MD simulations and experimental data on GB migration in pure metals. The activation energy for the GB migration obtained in the present study $(\sim 0.45 \mathrm{eV} /$ atom $)$ is smaller by a factor of $2-3$ than those typically measured experimentally. For example, the authors of [26] reported that the activation energy for GB migration in Ni is $1.18 \mathrm{eV} /$ atom. There are many possible contributions to this discrepancy (not mutually exclusive). It is useful to enumerate these. First, consider the possible sources of error in the simulation methods used to assess GB mobility:

- Of course, the interatomic potential used in any individual simulation study may be criticized. Certainly, the one employed in the present study has deficiencies (noted above), making direct comparisons with experiment subject to doubt. While this effect could contribute to the discrepancy, we believe that this is not the major source of error in comparison with experiment. For example, the value of the activation energy obtained in the present study is actually higher than the value $(0.25 \mathrm{eV} /$ atom $)$ obtained for the same GB in [27] using a different (and perhaps more realistic) interatomic potential. Foiles and Hoyt [13], using another zero-driving-force method, found an activation energy of $0.3 \mathrm{eV} /$ atom for the migration of a $\Sigma 7$ tilt GB in Ni (using a semi-empirical potential different from that used in the present study and in [27]). All of these activations energies are considerably smaller than experimentally measured values.

- In [11], it was pointed out that when driven MD methods are used, there is the potential that the applied driving force might be too high, and probe the non-linear portion of the $V-P$ relationship, or even the ballistic regime of GB motion. As [11] showed, this can indeed cause artifacts in extracted mobilities, and return artificially low activation energies for GB migration. Some, but certainly not all, driven motion simulations might suffer such artifacts. In the present study, where we have carefully verified that the GB motion is in the linear diffusional regime, we may rule this possibility out.

- MD simulations of individual boundaries tend to focus on a small set of high-symmetry grain boundaries - a situation that is rather idealized as compared with experiment. While some experimental papers quote activation energies for boundary migration extracted from grain growth experiments, this represents an ensemble average that is not strictly appropriate. This is an uncontrolled averaging procedure, heavily weighting the faster moving (lower activation energy) boundaries. To our knowledge, the connection between the mobilities of a few individual boundaries (such as studied in simulations) and the evolution of an entire grain boundary network (including triple junctions and other complications - such as texture effects) has not been rigorously established. While it 
is encouraging that for at least one specific simulated high-symmetry boundary with a single potential (a symmetric tilt $\Sigma 5 \mathrm{~GB}$ in Ni [11]) an activation energy close to the experimental range has been reported $(\sim 1.1 \mathrm{eV})$, no experimental data is available on the mobility of the same boundary. Simulations of all other GBs in Ni report lower activation energies-close to the one found in the present study.

Second, in addition to these simulation-related artifacts, there is of course a significant possibility of experimental issues:

- GB mobility in nominally pure metals can be significantly affected by impurity effects [28]. Impurities, in even trace quantities, are a concern in GB migration studies because of the effects of segregation, which tends to increase the impurity concentration at the GB (the concentration of impurities will tend to increase as the GB sweeps across the sample, picking up solutes). This extrinsic effect on grain boundary mobility measurements in experiments is inevitable, while MD simulations are generally performed in completely pure metals (without impurities). The applied strain method employed in the present paper was also employed in earlier work [12] to determine the activation energy for a nonsymmetric $\Sigma 5\langle 100\rangle \mathrm{GB}$ in Al. The activation energy was found to be $0.3 \mathrm{eV} /$ atom; half the experimental value ( $0.6 \mathrm{eV} / \mathrm{atom})$ for the same type of $\mathrm{GB}$ in $\mathrm{Al}$ [1] with only $0.5 \mathrm{ppm}$ of impurities (note, the experimental data were obtained using a curved boundary-i.e. one that samples a range of GB inclinations). More importantly, the mobility obtained from the simulations is two orders of magnitude larger than the experimental value for a sample containing only 8 ppm of impurities. Based on the analysis above and a re-examination of the data from [12], we believe that those simulations [12] were probing the correct, linear velocity-driving-force regime. For this case, then, the impurity drag effect seems to be a plausible explanation of the disagreement between the simulation and experimental GB mobility results, as discussed in [12].

As noted earlier, the discrepancy between the experimentally determined activation energies for grain boundary migration and those obtained from simulations may be attributable to more than one of the issues outlined above. Yet, the present results demonstrate that properly executed simulations of grain boundary migration do lead to dependable grain boundary mobilities (at least for the potentials and grain boundaries employed). It is our hope that the present effort at validating MD simulation approaches for measurement of grain boundary migration may offer a path toward the systematic study of these issues.

\section{Acknowledgments}

MIM gratefully acknowledges useful discussions with Dr D H Kim. Work at the Ames Laboratory was supported by the Department of Energy, Office of Basic Energy Sciences, under Contract No DE-AC02-07CH11358. CD was supported by the University of Manitoba, Canada. CAS acknowledges the support of the Solid State Solar Thermal Energy Conversion ( $S^{3}$ TEC) Energy Frontier Research Center, funded by the U.S. Department of Energy, Office of Science, Office of Basic Energy Sciences under DE-SC0001299.

\section{References}

[1] Gottstein G and Shvindlerman L S 1999 Grain Boundary Migration in Metals: Thermodynamics, Kinetics, Applications (Boca Raton, FL: CRC Press)

[2] Mishin Y, Asta M and Li J 2010 Acta Mater. 581117

[3] Schonfelder B, Wolf D, Phillpot S R and Furtkamp M 1997 Interface Sci. 5245 
[4] Zhang H, Mendelev M I and Srolovitz D J 2004 Acta Mater. 522569

[5] Janssens K G F, Olmsted D, Holm E A, Foiles S M, Plimpton S J and Derlet P M 2006 Nature Mater. 5124

[6] Olmsted D L, Foiles S M and Holm E A 2007 Scr. Mater. 571161

[7] Upmanyu M, Srolovitz D J, Shvindlerman L S and Gottstein G 1999 Acta Mater. 473901

[8] Zhang H, Upmanyu N and Srolovitz D J 2005 Acta Mater. 5379

[9] Molodov D A, Czubayko U, Gottstein G and Shvindlerman L S 1998 Acta Mater. 46553

[10] Mendelev M I and Srolovitz D J 2000 Acta Mater. 483711

[11] Deng C and Schuh C A 2011 Phys. Rev. B 84214102

[12] Mendelev M I, Srolovitz D J, Ackland G J and Han S 2005 J. Mater. Res. 20208

[13] Foiles S M and Hoyt J J 2006 Acta Mater. 543351

[14] Trautt Z T, Upmanyu M and Karma A 2006 Science 314632

[15] Deng C A and Schuh C A 2011 Phys. Rev. Lett. 106045503

[16] Lobkovsky A E, Karma A, Mendelev M I, Haataja M and Srojovitz D J 2004 Acta Mater. 52285

[17] Holm E A, Foiles S M, Homer E R and Olmsted D L 2012 Scr. Mater. 66714

[18] Mohles V and Zhou J 2012 Scr. Mater. 66717

[19] Ackland G J, Tichy G, Vitek V and Finnis M W 1987 Phil. Mag. A 56735

[20] www.ctcms.nist.gov/potentials/

[21] Morris J R and Song X Y 2002 J. Chem. Phys. 1169352

[22] ASM International Handbook Committee 1999 ASM Handbook (Materials Park, OH: ASM International)

[23] Monk J, Yang Y, Mendelev M I, Asta M, Hoyt J J and Sun D Y 2010 Modelling Simul. Mater. Sci. Eng. 18015004

[24] Supplementary data (stacks.iop.org/MSMSE/21/045017/mmedia)

[25] Plimpton S 1995 J. Comput. Phys. 1171

[26] Detor A J and Schuh C A 2007 J. Mater. Res. 223233

[27] Zhang H, Mendelev M I and Srolovitz D J 2005 Scr. Mater. 521193

[28] Cahn J W 1962 Acta Metall. 10789 